\title{
U-Shielder - an open source software for the estimation of gamma shielding using depleted uranium
}

\author{
J. Zeb ${ }^{*}$ and M. Wasim \\ Pakistan Institute of Nuclear Science and Technology (PINSTECH), P.O. Nilore, Islamabad, Pakistan.
}

Received: 24 February 2017 / Accepted: 27 September 2017

\begin{abstract}
U-Shielder is an open source software for the estimation of gamma-ray shielding using depleted uranium. It can be used to estimate thickness of shield for gamma-ray energies, ranging from $500 \mathrm{keV}$ to $10 \mathrm{MeV}$. The computational methodology is based on the point kernel technique. It uses exponential attenuation model with Taylor's form of build-up factor. Numerical method has been used to calculate shielding thickness. The software was developed in TURBO-C ++ and tested in both DOS and Windows operating systems. The software is available at Nuclear Energy Agency (NEA) website. The code proved useful in the designing of radioactive material transport container.
\end{abstract}

Keywords: U-Shielder / shielding / depleted uranium / build-up factor / simulation

\section{Introduction}

Gamma radiation possesses greater power of penetration than any other nuclear radiation except neutrons. While passing through matter, gamma rays interact with matter in different ways. Although more than ten distinct elementary processes have been identified by which gamma rays interact but for the purpose of radiation shielding only three processes are of any significance. These include photoelectric effect, compton scattering and pair production. Use of material to reduce intensity of gamma radiation is known as shielding (Nelson and Reilly, 1991). The effectiveness of a shielding depends on its thickness, material and energy of the radiation. Theoretically, all materials have some sort of radiation shielding. In practical situation, the choice of a shielding material dependents on many factors such as the final desired radiation levels, ease of heat dissipation, resistance to radiation damage, limitations on thickness and weight, multiple use considerations and availability of the material. It is known that higher the atomic number and density of a shielding material, the more effective it is in reducing intensity of gamma radiation (Al-Hamarneh, 2017).

Depleted uranium is considered as the best gamma-ray shielding material. Uranium is present everywhere: in soils, rocks and sea water. Its most common form is $\mathrm{UO}_{2}^{2+}$ (uraninite) and $\mathrm{U}_{3} \mathrm{O}_{8}^{2+}$ (pitchblende) (Betti, 2003; Bleise et al., 2003). The most abundant natural isotope of uranium is ${ }^{238} \mathrm{U}(99.2742 \%)$ followed by ${ }^{235} \mathrm{U}(0.7204 \%)$ and then ${ }^{234} \mathrm{U}$ $(0.0054 \%)$ (Rosman and Taylor, 1998). The amount of

\footnotetext{
*Corresponding author: zebe1968@yahoo.com
}

uranium remaining after the removal of enriched fraction is known as depleted uranium (DU), which typically contains about $99.8 \%$ (by mass) of ${ }^{238} \mathrm{U}, 0.2 \%$ of ${ }^{235} \mathrm{U}$ and $0.0006 \%$ of ${ }^{234} \mathrm{U}$ (Betti, 2003). DU has density of $19.1 \mathrm{~g} . \mathrm{cm}^{-3}$ that is $68 \%$ denser than lead. This property of DU has been exploited in designing radiation shielding containers, counter weights and ballasts. DU is five times more effective in gamma ray shielding than lead (Kok, 2009). Other uses of uranium include its application as catalyst, semiconductor and electrodes (Schlereth et al., 2005). The DU-based shielding materials greatly reduce the size of transport container or cask. However, the economic advantage gained through smaller cask equalizes the increased fabrication cost (Ferrada et al., 2004). Moreover, DU is slightly less toxic than lead (Yoshimura et al., 1995).

Since the emergence of nuclear technology, research in the field of shielding made a great progress that could be observed in the regular reviews published by Radiation Safety Information Computational Centre (RSICC) (Maskewitz et al., 1972). Even today new codes are being developed for new materials with different geometries (Subbaiah and Sarangapani, 2008). The main aim of this paper is to describe a shielding code, known as U-Shielder (NEA, 2012), which can be used to design DU based shielding.

\section{Description of the code}

U-Shielder has been developed to calculate shielding thickness of depleted uranium for gamma-rays originating from a point source at a minimum distance of $1 \mathrm{~cm}$. The computational methodology is point kernel technique (Subbaiah and Sarangapani, 2008) that calculates the gamma 


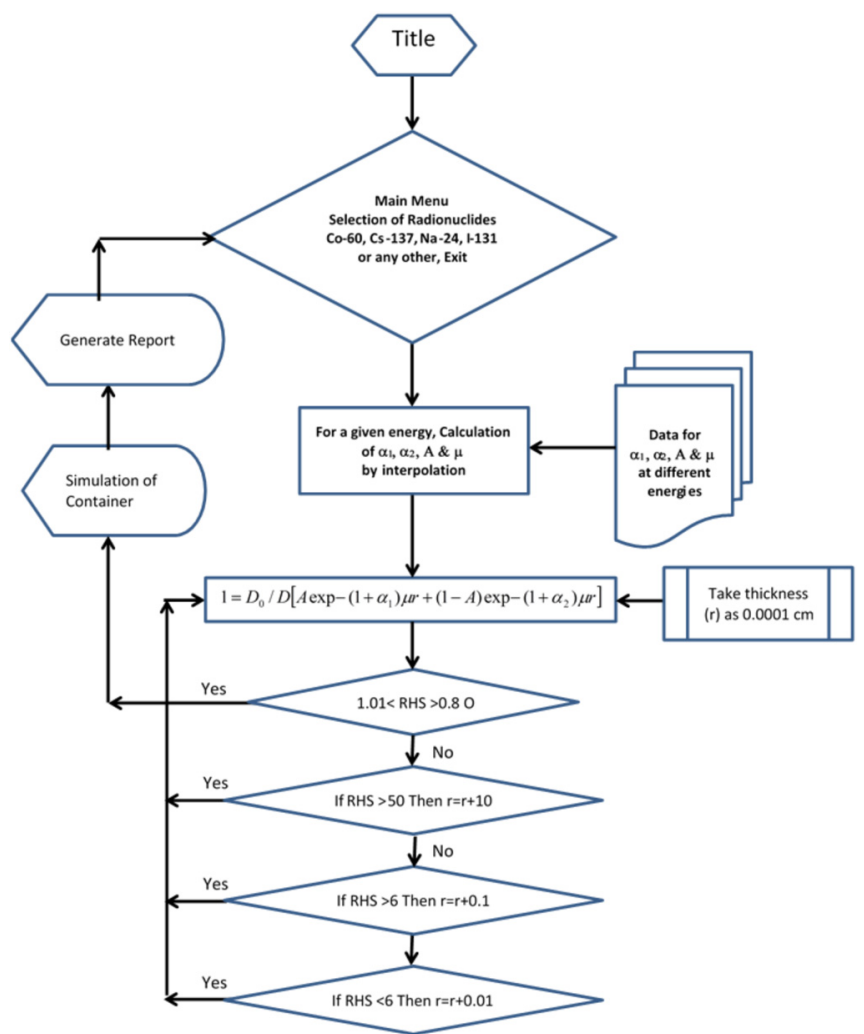

Fig. 1. U-Shielder algorithm for the optimization of various parameters.

ray build-up factor for a single layer shield using Taylor's formula (Harrison, 1958; Jaeger et al., 1968).

$$
B=A e^{\left(-\alpha_{1} \mu r\right)}+(1-A) e^{\left(-\alpha_{2} \mu r\right)}
$$

where A, $\alpha_{1}, \alpha_{2}$ are the Taylor build-up coefficients, $\mu$ is the linear attenuation coefficient and $r$ is the thickness of material. The first four parameters are taken from literature (Martin, 2006). The dose rate $D(r)$ at a distance $r$ from a point source, under the conditions of poor geometry, is given by (Cember and Johnson, 2008);

$$
D(r)=D_{0} e^{(-\mu r)} B
$$

After putting the value of $B$ from equation (1):

$$
\begin{aligned}
& D=D_{0}\left[A e^{-\left(1+\alpha_{1}\right) \mu r}+(1-A) e^{-\left(1+\alpha_{2}\right) \mu r}\right] \\
& 1=\frac{D_{0}}{D}\left[A e^{-\left(1+\alpha_{1}\right) \mu r}+(1-A) e^{-\left(1+\alpha_{2}\right) \mu r}\right]
\end{aligned}
$$

where $D_{0}$ is the dose rate or activity at $1 \mathrm{~cm}$ from the source.

Algorithm employed in the code is shown in Figure 1 as flow chart. It starts by taking input for radionuclide or energy, the activity $(\mathrm{mCi})$ or dose rate $\left(\mu \mathrm{Sv}_{\mathrm{h}} \mathrm{h}^{-1}\right)$ of the source and desired dose rate $\left(\mu \mathrm{Sv} \cdot \mathrm{h}^{-1}\right)$ after the DU shield. For a given energy, the values of $A, \alpha_{1}, \alpha_{2}$ and $\mu$ are calculated by interpolation using data files. These values along with an initial value of $r=0.0001$ are used in equation (4). U-Shielder optimizes the value of $r$ for the desired dose rate. After

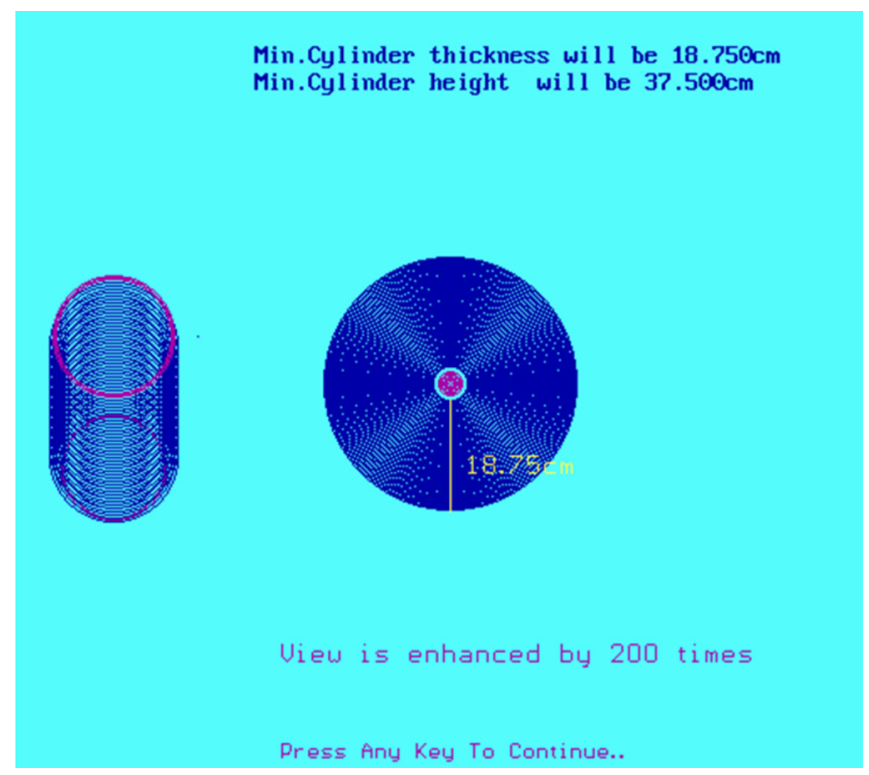

Fig. 2. U-Shielder simulating the shielding after estimation.

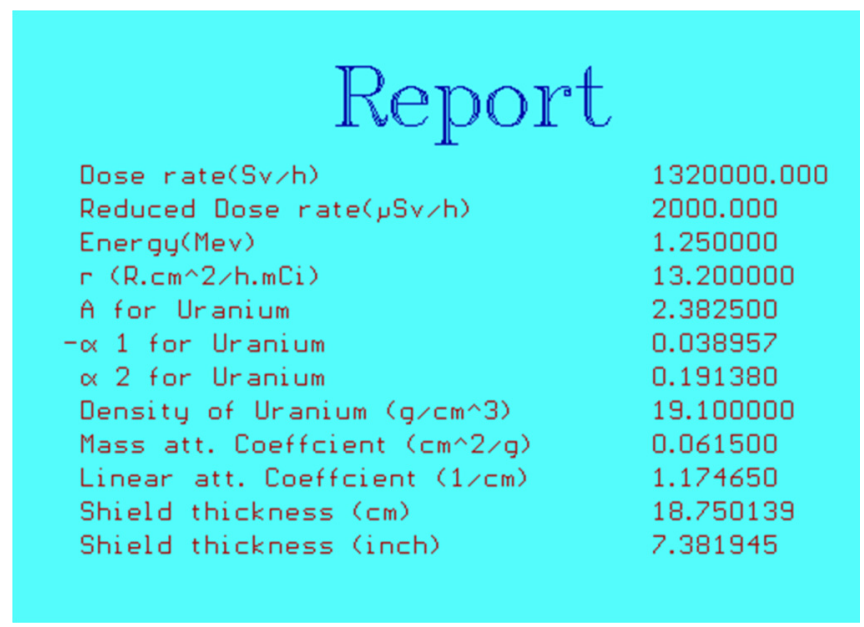

Fig. 3. Report generated by U-Shielder after estimating shielding.

estimating the shielding thickness, U-Shielder creates a simulation for the desired thickness and height. The view of plotted figures are enhanced or reduced accordingly. The simulation created by U-Shielder for the shielding estimation for ${ }^{60} \mathrm{Co}$ source having activity $3.7 \times 10^{14} \mathrm{~Bq}$ and desired dose rate of $2 \mathrm{mSv} \cdot \mathrm{h}^{-1}$ is exhibited in Figure 2. The report generated by U-Shielder is shown in Figure 3.

The general features of U-Shielder include its capability to estimate shielding thickness for:

- photons in energy range 0.5 to $10 \mathrm{MeV}$;

- input as activity or dose rate;

- desired dose rate after the shielding.

The results of U-Shielder were compared with the data available in literature (Sauermann, 1976). A comparison of UShielder and literature values is presented in Figure 4. The comparison revealed variation of relative error from $+4.4 \%$ to $-7.6 \%$ for all the attenuation factors. 


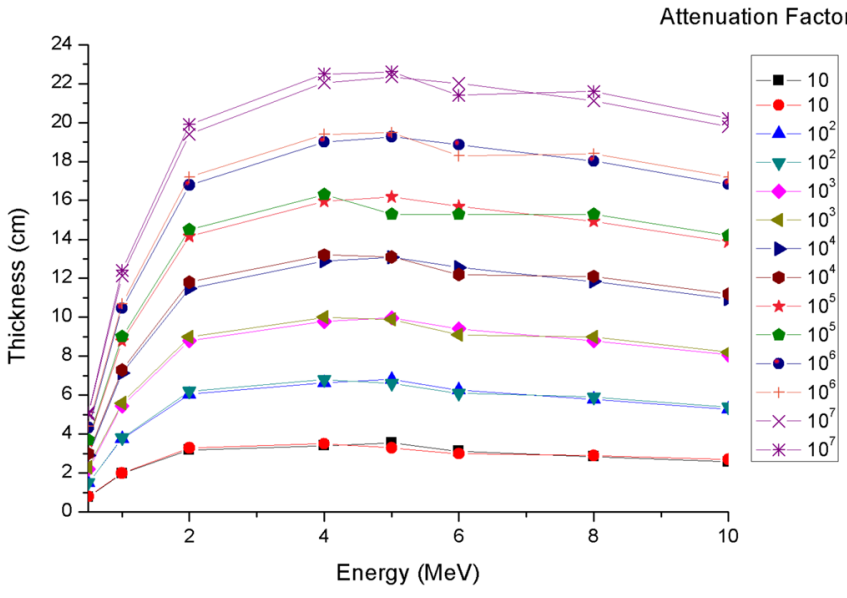

Fig. 4. Comparison of U-Shielder results with the literature data (Sauermann, 1976) for different gamma energies and attenuation factors.

Table 1. DU thickness $(\mathrm{cm})$ estimated by U-Shielder to shield the radionuclides for safe transportation.

\begin{tabular}{lrrrrr}
\hline Radionuclide & \multicolumn{5}{c}{ Activity (Bq) } \\
\cline { 2 - 6 } & $3.7 \times 10^{10}$ & $3.7 \times 10^{11}$ & $3.7 \times 10^{12}$ & $3.7 \times 10^{13}$ & $3.7 \times 10^{14}$ \\
\hline${ }^{137} \mathrm{Cs}$ & 4.46 & 5.47 & 6.48 & 7.48 & 8.49 \\
${ }^{60} \mathrm{Co}$ & 10.57 & 12.62 & 14.67 & 16.71 & 18.75 \\
${ }^{131} \mathrm{I}$ & 4.79 & 5.92 & 7.04 & 8.16 & 9.28 \\
${ }^{99} \mathrm{Mo}$ & 4.62 & 5.78 & 6.94 & 8.10 & 9.26 \\
\hline
\end{tabular}

The software displays results in the form of a report, which includes the estimated thickness in $\mathrm{cm}$ or inch, interpolated values of the three parameters for Taylor build-up coefficients, and mass attenuation coefficient of depleted uranium for the desired energy. The processing time of U-Shielder is about 15 to 30 seconds, depending upon the speed of computer and attenuation required. The software is available for free from NEA Data Bank with the ID IAEA 1434/01 (NEA, 2012). The package contains executable file, source codes and user manual.

U-Shielder has been employed to estimate the shielding for different radionuclides $\left({ }^{60} \mathrm{Co},{ }^{137} \mathrm{Cs},{ }^{131} \mathrm{I},{ }^{99} \mathrm{Mo}\right)$ commonly used in nuclear industry and oncology centers for different activities (Tab. 1). The calculations were made for considering the desired dose rate of $2 \mathrm{mSv} \cdot \mathrm{h}^{-1}$ at the surface of container for safe transportation (IAEA, 2009).

\section{Conclusions}

U-Shielder is a user-friendly software with graphical user interface for the calculation of shielding using depleted uranium. It calculates thickness of shielding for gamma rays having energy in the range of $500 \mathrm{keV}$ to $10 \mathrm{MeV}$. The software operates both in DOS and Windows environment. Code validation showed maximum relative deviation of less than 8\%. Nuclear Energy Agency (NEA) released the U-Shielder on its website after verifying its results. The code has been used for estimating shielding at various laboratories of PINSTECH. It revealed to be a stable and reliable code.

\section{References}

Al-Hamarneh IF. 2017. Investigation of gamma-ray shielding effectiveness of natural marble used for external wall cladding of buildings in Riyadh, Saudi Arabia. Results in Physics 7: 792-798.

Betti M. 2003. Civil use of depleted uranium, J. Environ. Radioact. 64: 113-119.

Bleise A, Danesi P, Burkart W. 2003. Properties, use and health effects of depleted uranium (DU): a general overview, J. Environ. Radioact. 64: 93-112.

Cember H, Johnson TE. 2008. Introduction to Health Physics. 4th edition, NY: McGraw-Hill.

Ferrada JJ, Mattus CH, Dole LR. 2004. Radiation shielding using depleted uranium oxide in nonmetallic matrices. Oak Ridge: Oak Ridge National Laboratory (ORNL).

Harrison JR. 1958. Nuclear reactor shielding. New York: Temple Press.

Jaeger RG, Blizard E, Chilton A, Grotenhuis M, Hoenig A, Jaeger TA, Eisenlohr H. 1968. Engineering compendium on radiation shielding. Volume I. Shielding fundamentals and methods. New York: Springer-Verlag.

Kok KD. 2009. Nuclear engineering handbook. Boca Raton: CRC Press.

Martin JE. 2006. Physics for radiation protection: a handbook. Mörlenbach: John Wiley \& Sons.

Maskewitz BF, Clark FH, Trubey D. 1972. Computer codes for shielding and related calculations-1972, Nucl. Eng. Des. 22: 334-341.

NEA, 2012. IAEA1434 U-SHIELDER. U-SHIELDER, Estimates shielding thickness of depleted uranium for photons from 0.5 to 10 MeV. http://www.oecd-nea.org/tools/abstract/detail/iaea1434/

Nelson G, Reilly D. 1991. Gamma-ray interactions with matter. Passive non-destructive analysis of nuclear materials. Los Alamos: Los Alamos National Laboratory (LANL), pp. 27-42.

Rosman K, Taylor P. 1998. Isotopic compositions of the elements 1997 (Technical Report), Pure Appl. Chem. 70: 217-235.

Sauermann P-F. 1976. Radiation protection by shielding. Tables for the calculation of gamma radiation shielding. Muenchen: Karl Thiemig, Vol. 11, 191 p. ISBN : 3521061027

Schlereth T, Hedhili M, Yakshinskiy B, Gouder T, Madey T. 2005. Adsorption and reaction of $\mathrm{SO}_{2}$ with a polycrystalline $\mathrm{UO}_{2}$ film: promotion of SO bond cleavage by creation of O-defects and $\mathrm{Na}$ or Ca co-adsorption, J. Phys. Chem. B 109: 20895-20905.

Subbaiah K, Sarangapani R. 2008. IGSHIELD: a new interactive point kernel gamma ray shielding code. Ann. Nucl. Energy 35: 2234-2242.

Yoshimura H, Gildea P, Bernard E. 1995. Using depleted uranium to shield vitrified high-level waste packages. Sandia National Labs., Albuquerque, NM (United States). Funding organisation: USDOE, Washington, DC (United States).

Cite this article as: Zeb J, Wasim M. 2017. U-Shielder - an open source software for the estimation of gamma shielding using depleted uranium. Radioprotection 52(4): 273-275 